\title{
Resiliencia en estudiantes exitosos en matemáticas
}

Martha Cecilia Santiago Carrillo ${ }^{1}{ }^{\circledR}$, , Henry de Jesús Gallardo Pérez ${ }_{\text {Universidad Francisco de Paula Santander-Colombia }}^{\circledR}$

Autor de correspondencia:

${ }^{1}$ marthacecilia@ufps.edu.co

Recibido: 06 de septiembre de 2019 Revisado: 10 de noviembre de 2019 Aprobado: 20 de febrero de 2020 Publicado: 24 de mayo de 2020

\section{Resumen}

El objetivo de investigación fue evaluar la relación entre el rendimiento académico en matemáticas y el éxito escolar en estudiantes de educación superior. La investigación siguió un enfoque cuantitativo, descriptivo-comparativo y microsociológico. Los datos provinieron de fuentes primarias. La recolección de datos fue transeccional y la muestra fue de 1500 estudiantes. Se utilizó la escala de resiliencia de Wagnild y Young. Los resultados muestran que hay una relación importante entre el nivel de exigencia, la aplicación de las matemáticas, la abstracción y el rendimiento académico. Los estudiantes desarrollan habilidades emocionales de resiliencia que les permiten mejorar su calidad de vida. En conclusión, la resiliencia se relaciona significativamente con todas las fortalezas del carácter, así como con la aceptación positiva del cambio y con la competencia personal. Se identificó que, si bien la resiliencia ayuda a los estudiantes a ser más tolerantes y a comprender mejor lo que sucede en el entorno, es precisamente en esos desafíos donde se manifiestan las fortalezas que les brindan un alto desempeño educativo. Los estudiantes exitosos demuestran habilidades de pensamiento formal, pensamiento creativo, aplicación de la matemática y resiliencia, las cuales inciden en su calidad de vida.

Palabras clave: matemáticas, éxito escolar, rendimiento académico, resiliencia, calidad de vida 


\title{
Resilience in successful math students
}

\begin{abstract}
The research objective was to assess the relation between academic performance in mathematics and school success in higher education students. The research adopted a quantitative, descriptive-comparative and microsociological approach. Data came from primary sources. Data collection was transectional and the sample consisted of 1500 students. The Wagnild and Young's resilience scale was used. The results show that there is a strong relation among the exigency level, the application of mathematics, abstraction, and academic performance. Students develop emotional resilience skills that allow them to improve their quality of life. In conclusion, resilience is significantly related to all personality strengths, as well as to positive change acceptance and personal competence. It was identified that, while resilience helps students become more tolerant and better understand what is happening in their environment, it is precisely in these challenges where the strengths brought by high educational performance are manifested. Successful students display formal thinking skills, creative thinking, application of mathematics, and resilience, all of which influence their quality of life.
\end{abstract}

Keywords: mathematics, school success, academic performance, resilience, quality of life

\section{Resiliência em estudantes bem-sucedidos em matemáticas}

\section{Resumo}

O objetivo da pesquisa foi avaliar a relação entre o desempenho acadêmico em matemáticas e o bom sucesso escolar em estudantes do ensino superior. A pesquisa seguiu uma abordagem quantitativa, descritiva-comparativa e micro-sociológica. Os dados são provenientes de fontes primárias. A colheita de dados foi transversal e a amostra foi de 1500 estudantes. Utilizou-se a escala de resiliência de Wagnild e Young. Os resultados mostram que há uma relação importante entre o nível de exigência, a aplicação das matemáticas, a abstração e o desempenho acadêmico. Os estudantes desenvolvem habilidades emocionais de resiliência que lhes permitem melhorar sua qualidade de vida. Em conclusão, a resiliência está relacionada significativamente com todas as fortalezas de caráter, assim como com a aceitação positiva da mudança e com a competência pessoal. Identificou-se que, embora a resiliência ajude aos estudantes ser mais tolerantes e a compreender melhor o que acontece no entorno, é precisamente naqueles desafios onde se manifestam as fortalezas que lhes dão um alto desempenho educacional. Os estudantes bem-sucedidos demonstram habilidades de pensamento formal, pensamento criativo, aplicação da matemática e resiliência, que incidem em sua qualidade de vida.

Palavras-chave: matemática, bom sucesso escolar, desempenho acadêmico, resiliência, qualidade de vida 


\section{Introducción}

El éxito escolar es una noción que ha cambiado según la evolución de las corrientes pedagógicas. "Desde un enfoque humanista, el rendimiento académico es el producto que da el alumnado en los centros de enseñanza y que habitualmente se expresa a través de las calificaciones escolares" (Duek, 2010). El aspecto más visible del rendimiento son las calificaciones, que son analizadas principalmente desde dos enfoques: el cuantitativo -que tiene matices conductistas y cognitivistas - y el cualitativo - donde se identifican los estilos de aprendizaje, predisposiciones y características personales del estudiante-. Este último tiene una mirada más humana.

En el contexto de la investigación, en el rendimiento se reconoce la influencia de los factores sociales, pues allí hay elementos que impulsan y moldean su desarrollo en las aulas de clase. En ese sentido, no se ve al estudiante como una entidad aislada que se autodetermina, sino como el resultado de estímulos originados en "la diversidad de factores que inciden en el rendimiento, [...] este depende no solo de las aptitudes intelectuales, sino de una serie de factores interrelacionados, tanto internos como externos al estudiante" (Hallinger \& Heck, 2014), lo cual requiere una visión más profunda y completa de la escuela como el escenario donde las personas adquieren de manera recíproca sus pautas sobre liderazgo y convivencia. En su visión se debería asignar la misma importancia a las habilidades sociales como se hace con los conocimientos teóricos tradicionales. No obstante, los centros educativos tienden a definir un horizonte de acción orientado hacia el logro y el cumplimiento de metas de aprendizaje que configuran un clima de convivencia escolar específico. El estructural funcionalismo plantea que en el sistema escolar se enseña, además de los contenidos teóricos o técnicos, valores y formas de entender el mundo, reglas sociales, como el hecho de vivir en una sociedad regida por la competencia (Rodríguez \& Valdivieso, 2008)

La interrelación se presenta como la clave para analizar el rendimiento, ya que permite ver cómo se ponen en común los aspectos vinculados con la experiencia educativa y cómo se identifican los principales elementos que determinan el contexto de una institución y de un grupo en específico. Existen dos corrientes que se contraponen en su comprensión: por una parte, el conductismo destaca que "hay aprendizaje cuando hay un cambio conductual; en tanto conducta observable e identificable" (Pérez, 2012); mientras que el cognitivismo se enfoca en los elementos que producen las funciones cognitivas en el cerebro - donde intervienen ideas, pensamientos, planes, memoria y la capacidad de aprendizaje (García, 2003)-. En este sentido, todos los seres humanos estamos expuestos a situaciones dolorosas, estresantes, difíciles en la vida. Algunas personas colapsan y se desequilibran; otras transforman sus debilidades, asumen su potencialidad y hasta resultan airosas y fortalecidas por esas mismas circunstancias. Diferentes investigadores han analizado y probado el vínculo entre el éxito escolar y la motivación personal. Algunos, como García (2008), precisan su naturaleza multidimensional, donde entran en juego variables afectivas y cognitivas, que en su interacción contribuyen a formar la valoración que tiene el estudiante de sí mismo y de su entorno (García, 2003). Reconocer esta complejidad ha generado guías y clasificaciones, como la formulada por Covington (2000) en su modelo de autovaloración, en el que identifica estudiantes orientados al dominio, cuyos principales intereses son el rendimiento y la motivación. También están los que evitan el fracaso y por eso no participan 
de la vida escolar, además de los que aceptan el fracaso, quienes asumen una actitud derrotista y de bajo esfuerzo (Castro, 2010). No obstante, ninguna de esas decisiones es resultado de un ambiente homogéneo.

Frente al interrogante del impacto del éxito académico en las relaciones interpersonales, surgen varias situaciones, como el hecho de que haya jóvenes que se comportan y socializan para seguir siendo considerados los mejores de la clase, mientras que hay otros que enfrentan dificultades para mantener el ritmo. Entre tanto, algunos se dedican a ridiculizar a quienes tienen éxito académico. Este espectro de actitudes frente a la experiencia educativa influye en la calidad de las relaciones interpersonales que allí se gestan. Es un tema que se retroalimenta de forma permanente, pues el sentirse aceptado o excluido es considerado un factor psicosocial que puede determinar el rendimiento de los estudiantes (Cruz, 2016).

Dado que estos aspectos están estrechamente relacionados, en el ambiente de aula se observa cómo los mismos compañeros, con calificaciones similares, deficientes en matemáticas - por definir un área de análisis—, se reúnen siempre para desarrollar trabajos grupales. Mientras tanto, los que entienden mejor los temas son apartados. Esa polarización social configura fenómenos de exclusión que no favorecen los procesos de enseñanzaaprendizaje. Tampoco benefician a las familias ni a las instituciones de educación, puesto que los jóvenes desarrollan temores, limitaciones y debilidades que se proyectan en su personalidad y en sus habilidades para relacionarse de forma asertiva, lo cual incluso los lleva a desertar de la etapa de formación.

Cuando los estudiantes excelentes se convierten en jóvenes aislados en un grupo social y son estigmatizados por su condición de ser buenos, se evidencian las debilidades del desarrollo integral de los aprendizajes y habilidades. Así pues, es preciso evaluar hasta qué punto el éxito académico permite afrontar mejor las situaciones y solucionar problemas interpersonales (Mata, Gómez \& Calero, 2016).

Ahora bien, la caracterización del estudiante exitoso está, en cierta forma, relacionada con la escolaridad. Así, se encuentra que los jóvenes que cursan los primeros semestres muestran relaciones interpersonales más satisfactorias, mientras que quienes van más adelante tienen más dificultades para lograr mejores experiencias de interacción con grupos de amigos y para emplear sus logros académicos dentro de los ambientes sociales. En lo cotidiano, los educandos priorizan la oportunidad de construir vínculos con sus compañeros, aunque estos resulten volátiles y cambiantes, puesto que asumen la institución educativa como un espacio donde la convivencia determina sus logros y obstáculos para alcanzar los objetivos propuestos. Sin embargo, cuando se ven excluidos de esa dinámica, reducen sus actitudes y habilidades de competencia social (Rivera, Gallardo, Mora, Gómez \& Barrios, 2018).

Otro factor importante en el rendimiento escolar del alumno es la motivación, la cual es un proceso general por el que se inicia y se dirige una conducta hacia el logro de una meta. Motivar a los estudiantes implica fomentar sus recursos internos, sentido de competencia, autoestima, autonomía y realización. Se necesita abordar el éxito académico según los diferentes factores y entender cómo se articulan bajo una lógica emocional, puesto que las emociones mediatizan la capacidad de adaptarse y de responder a una variedad de experiencias, al tiempo que preparan al organismo para responder rápidamente a las amenazas del mundo circundante (Jadue, 2002). Asimismo, los psicólogos sociales plantean 
que las emociones humanas son fundamentales para el apego, la interacción y la función social (Sierra, 1989)

Reconocer el papel de las emociones es un punto de partida invaluable para este proyecto de investigación, porque permite correlacionar el rendimiento académico con factores que suelen ser ignorados y que en ocasiones se piensa que no tienen sustento teórico/científico.

Wagnild y Young (1993) proponen una escala de 25 ítems, entre 25 y 175 puntos en torno a la resiliencia (Olivera, Braum \& Roussos, 2011), entendida como el rasgo positivo de personalidad que permite a los sujetos adaptarse a las adversidades de la vida (Mattelart, 2003), al enfrentarlas con valentía y reducir, por lo tanto, el efecto nocivo del estrés (García, 2008). La resiliencia resulta de factores protectores como autoestima consistente, introspección, independencia, capacidad para relacionarse, iniciativa, humor, creatividad, moralidad y pensamiento crítico (Kotliarenco, Cáceres \& Fontecilla, 1997). En la perspectiva de Grotberg (2006), estas fuentes se pueden clasificar en tres categorías: 1) aquellas que tienen que ver con el apoyo que la persona cree que puede recibir - yo tengo—; 2) aquellas que tienen que ver con las fortalezas intrapsíquicas y condiciones internas de la persona yo soy, yo estoy- (Moreno, 2011); y 3) aquellas que tienen que ver con las habilidades de la persona para relacionarse y resolver problemas - yo puedo- (Kotliarenco, 1999). Por su parte, Saavedra (2003) desarrolla un modelo emergente del estudio de casos, donde señala que la respuesta resiliente es una acción orientada a metas. Dicha respuesta se sustenta o se vincula con una visión abordable del problema. Como conducta recurrente, la visión de sí mismo se caracteriza por elementos afectivos y cognitivos positivos o proactivos ante los problemas, los cuales tienen como condición histórico-estructural las condiciones de base, es decir un sistema de creencias y vínculos sociales que impregnan la memoria de seguridad básica y que, de modo recursivo, interpreta la acción específica y los resultados.

En el ámbito psicosocial, se diferencian los factores psicológicos de los factores sociales y culturales. En los primeros se define la pertinencia del espacio o lugar de estudio, la organización del tiempo colectivo y personal, así como las técnicas de estudio empleadas. En el segundo grupo se establece la clase social, el clima de convivencia y la influencia familiar.

El éxito escolar no siempre es motivo de regocijo, debido a que no todos sus efectos son positivos. A pesar de que generalmente sea visto de esa manera - suele creerse que "tener éxito, en el ámbito educativo es la consecución de los objetivos marcados en un plan de estudios" (Osorio, Mejía \& Navarro, 2009)—, esta investigación es pertinente, pues se propone evaluar cómo el rendimiento impacta las relaciones interpersonales, para establecer hasta qué punto el desarrollo de habilidades excepcionales e integrales puede apoyar la resolución de conflictos sociales por parte de los estudiantes víctimas de acoso o exclusión.

En el presente estudio se profundiza en los diferentes factores académicos, culturales y socioeconómicos que influyen no solo en las calificaciones obtenidas, sino también en las formas de relacionamiento que caracterizan a los jóvenes, al considerar que deben enfrentar retos complejos de aprendizaje en áreas como las matemáticas. Es necesario combinar todos esos aspectos de la realidad educativa para hallar información confiable que permita comprender mejor este tema. 
El problema se plantea desde el contexto universitario. Se toman específicamente los estudiantes de los primeros semestres. Se quiere revisar cómo son los ambientes de convivencia con sus demás compañeros, además de identificar características realistas que conduzcan a establecer el papel del éxito académico dentro de las interacciones sociales originadas en la experiencia educativa.

Se parte de la idea de que al ignorar las consecuencias que produce el rendimiento excepcional en la vida cotidiana de los estudiantes, se desaprovecha la oportunidad de profundizar en su experiencia formativa y de caracterizar los factores involucrados en su desarrollo personal. De tal forma, este proyecto de investigación propone identificar las actitudes, comportamientos y factores, así como las correlaciones que subyacen y determinan la dinámica social.

Con el propósito de armar el rompecabezas completo que implica la convivencia actual en la institución educativa, se propone derribar las apariencias de las calificaciones, los números y las letras, para conocer cómo se sienten realmente los jóvenes estudiantes en los procesos de enseñanza y de aprendizaje y cómo consideran que sus expectativas de excelencia personal afectan o contribuyen en su capacidad de tener relaciones de calidad con sus compañeros, al tiempo que inciden en las prácticas pedagógicas de sus docentes. El estudio busca impactar en la dimensión humana del estudiante, puesto que, en su experiencia, los investigadores han identificado que, solo a partir de una relación sana consigo mismos y con el entorno, los jóvenes logran superar barreras y trascender la formación académica, al convertirla en formación para la vida. Como aporte, por medio de la investigación, se ofrece una forma de concretar una visión profesional que oriente la atención sobre la complejidad de los procesos de enseñanza y de aprendizaje, al analizarlos desde nuevos puntos de vista que consideren los impactos que causan en la vida de los estudiantes. Esta experiencia puede ser un precedente para una línea de investigación sobre los efectos que la presión académica genera en los jóvenes.

\section{Método}

La investigación se enmarca en un enfoque multimétodo (Gallardo, Vergel \& Villamizar, 2018) de carácter cuantitativo y descriptivo-comparativo, según las características del objeto de estudio y el contexto en que se desarrolla. Los datos provinieron de fuentes primarias. Desde el punto de vista cuantitativo, el enfoque es descriptivo y transeccional, además de seguir el método correlacional (Sierra, 1989). Por otro lado, desde el enfoque cualitativo, la investigación se basa en el método etnográfico y microsociológico, el cual permite abordar el problema del estudio desde tres perspectivas - todas de gran utilidad-: como enfoque, permite comprender las situaciones al considerar el punto de vista de las personas involucradas; como método, motiva a ir al terreno donde ocurren los eventos; y como texto, permite describir de manera textual los hallazgos y comportamientos (Grotberg, 2006), y aborda la naturaleza del problema, las experiencias personales de los principales actores, sus actitudes y prácticas, así como las características del contexto en el que se produce, en cuyo interior están los instrumentos básicos para un mejor análisis e interpretación. Todo ello permite garantizar la confianza en los resultados como fiel representación de lo que sucede con el fenómeno estudiado. Desde la perspectiva holística, ver el escenario y a las personas 
no reducidas a variables, sino consideradas como un todo integral, que obedece a una lógica de organización de la institución, funcionamiento y significado, permite entender los acontecimientos a partir de las múltiples interacciones que los caracterizan. Desde la perspectiva naturalista, el enfoque está en la lógica interna de la realidad que se analiza.

La investigación tiene un diseño secuencial, con un grupo de 1500 estudiantes de diferentes programas académicos que cursan de primer a cuarto semestre en instituciones de educación superior en Norte de Santander, Colombia, y que se han matriculado al menos en un curso de matemáticas. El material utilizado en la investigación proviene de la aplicación de la escala de resiliencia de Wagnild y Young (2015), un cuestionario autoadministrado que se desarrolla en torno a dos factores:

- Competencia personal -17 ítems-: autoconfianza, independencia, decisión, invencibilidad, poderío, ingenio y perseverancia.

- Aceptación de uno mismo y de la vida -8 ítems-: adaptabilidad, flexibilidad, balance y perspectiva de vida estable.

Los factores están representados en cinco áreas de la resiliencia: satisfacción personal -4 ítems - , sentirse bien solo -3 ítems - , confianza en sí mismo -7 ítems-, ecuanimidad -4 ítems - y perseverancia -7 ítems - . El estudio se hizo con coeficientes de correlación ítem-test corregido o índice de discriminación mediante el coeficiente de correlación Pearson -entre 0.61 y 0.89 estadísticamente significativos-, los resultados del coeficiente alfa global — nivel de fiabilidad de 0.8 - y la proporción de variación de los factores (Crombie, López, Mesa \& Samper, 2015; Sánchez \& Robles, 2015). Esta escala ha sido utilizada en un buen número de investigaciones, tesis doctorales y proyectos de maestría. Finalmente, se propuso la triangulación de los hallazgos obtenidos a través de las diferentes técnicas de recolección.

\section{Resultados}

El análisis cualitativo muestra que el éxito académico de los jóvenes está asociado con categorías motivacionales influenciadas desde la familia; con aspectos psicológicoso sociales, desde compañeros de clase y personas cercanas; y con la cultura como condicionante del clima de convivencia, la empatía y la autoestima. También surge la clase social como un factor en instituciones privadas. Bajo este punto de vista, el estudiante no es una presencia aislada, sino la suma de diferentes influencias que lo llevan a comportarse de cierta manera y a elegir ciertas prioridades. Los diferentes factores identificados están relacionados - y se expresan a diario- con la importancia de que las personas crean en lo que se les promete, la aceptación positiva del cambio, la competencia personal, el control y la espiritualidad. Estos factores explican el 5.39\% de la variabilidad total de los datos.

El análisis cuantitativo muestra una consistencia interna de los factores. Se realizó mediante el modelo alfa de Cronbach. Se obtuvo una escala total de resiliencia de 0.87. En las subescalas se obtuvo: 0.86 en aceptación positiva del cambio, 0.82 en competencia personal, 0.72 en control y 0.32 en espiritualidad. De otra parte, los resultados de la prueba de resiliencia indican que se dan puntuaciones más bajas en los puntos 6 -en situaciones de emergencia, me siento agresivo e incómodo-, 24 -tiendo a perder el control durante las 
emergencias - y 27 - cuando veo a alguien que necesita ayuda urgente en una emergencia, me desmorono-- El punto 6 registró un promedio de 2.12, que corresponde a la subescala de sufrimiento personal. El punto 24 tuvo un promedio de 2 también en la subescala de sufrimiento personal. El punto 27 alcanzó un promedio de 2.07, y forma parte de la misma escala mencionada atrás. Los ítems con mayor puntuación fueron el 8 -trato de tomar en cuenta a cada una de las partes (opiniones) en un desacuerdo (conflicto) antes de tomar una decisión-y el 18 -cuando veo a alguien que está siendo tratado injustamente, a veces no siento compasión por él一. El ítem 8 registró un promedio de 4.32 en la subescala de toma de perspectiva. El ítem 18 tuvo un promedio de 4.15, lo cual es parte de la subescala de preocupación empática (tabla 1).

Tabla 1

Análisis descriptivo - Tendencia por item

\begin{tabular}{ll}
\hline ÍTEM & Media \\
\hline Adaptación a cambios & 4.57 \\
Confío en las personas & 3.02 \\
Resuelvo conflictos & 4.15 \\
Veo el lado positivo de las cosas & 3.65 \\
Me recupero rápido de dificultades & 3.97 \\
No abandono lo que tiende a no tener solución & 3.90 \\
Tomo iniciativa frente a solución de problemas & 3.70 \\
Me animo ante el fracaso & 3.97 \\
Pensamiento positivo soy fuerte & 3.60 \\
Manejo sentimientos desagradables & 2.77 \\
Agrado por los desafíos & 3.32 \\
Alcanzo las metas propuestas & 4.20 \\
Claridad hacia dónde proyecto mi vida & 4.60 \\
Trabajo para conseguir metas & 3.35 \\
Me siento orgulloso de mis triunfos & 3.95 \\
Tomo decisiones difíciles o impopulares & 3.50 \\
Afronto el estrés & 3.80 \\
Bajo presión me concentro & 3.30 \\
Control de vida & 4.15 \\
Sigo presentimientos & 2.97 \\
Dejo al destino, ayuda de Dios, las situaciones & 2.12 \\
Las situaciones ocurren por alguna razón & 2.60 \\
Escucho consejos & 2.30 \\
Apoyo a quien tiene dificultades & 2.82 \\
Ante la adversidad me desanimo & 2.47 \\
\hline & \\
\hline
\end{tabular}

Fuente: elaboración propia.

Los resultados de las cuatro subescalas evaluadas muestran que la puntuación más alta está asociada a la resiliencia respecto a la dimensión proyecto de vida y aceptación positiva al cambio -27, alta-, mientras que la puntuación más baja se registró en las dimensiones confiar en Dios y apoyar al otro. En general, las dimensiones arrojaron una evaluación normal. Solo la dimensión de resiliencia se ubica en el nivel alto. En cuanto a las cuatro 
subcategorías principales que establece el instrumento, en la empatía cognitiva, formada por las subescalas de perspectiva y fantasía, se ha encontrado una disposición consistente de los alumnos a ponerse en el lugar del otro y a sentirse identificados por las experiencias que consumen a través de los medios de comunicación o el entretenimiento. En la subcategoría de empatía afectiva, que involucra las subescalas de preocupación y sufrimiento, se refleja una menor disposición a sentirse incómodo o ansioso con las malas experiencias, en comparación con lo que sus compañeros pueden experimentar, en especial cuando observan directamente las situaciones de estrés o conflicto. En general, en los reactivos se establece una normalidad en la resiliencia total de 90 en edades entre 17 a 21 años y en la empatía emocional de 41 - de una posible puntuación ideal de 70-. Esto revela una alta categoría cognitiva y afectiva, razón que lleva a establecer una normalidad en la medida global de empatía con una puntuación media de 83.9 frente a una posible puntuación de 40 .

Tabla 2

Frecuencias estadísticas por subescala

\begin{tabular}{lllll}
\hline Estadísticos & R1 & R2 & R3 & R4 \\
\hline Average & 84,9250 & 88,9250 & 84,6000 & 76,4000 \\
Medium & 85,0000 & 80,0000 & 85,0000 & 75,5000 \\
Fashion & 83,00 & 80,00 & 82,00 & 83,00 \\
Desv. Desviation & 3,56901 & 10,02803 & 3,37259 & 8,19890 \\
\hline
\end{tabular}

Fuente: elaboración propia.

En la tabla 2 se presentan los resultados de la media correspondiente a las cuatro subescalas evaluadas por el Test Davis, donde el mayor puntaje se encuentra en la dimensión competencia personal con 88,9 , mientras que el menor puntaje se registró en la dimensión espiritualidad con 76,4. Sin embargo, la dimensión con mayor puntaje es también la que presenta mayor variabilidad, lo que indica altas diferencias en el rango; por el contrario, los puntajes en las dimensiones aceptación positiva al cambio y control, aun cuando no son los mayores, sí son bastante homogéneos. También resulta relevante que la dimensión espiritualidad no solo tiene el menor puntaje, sino que además los valores presentan una alta variabilidad.

Tabla 3

Resultados generales en resiliencia

\begin{tabular}{lll}
\hline Sub-Escala & Resultados & Rating \\
\hline Seguridad personal (RS1) & 78.5 & Normal \\
Aceptación positiva al cambio (R1) & 84,9 & Normal \\
Competencia personal (R2) & 88,9 & Alto \\
Autoestima (RS3) & 70.5 & Normal \\
Control (R3) & 84,6 & Normal \\
Afiliación (RS2) & 71 & Normal \\
Altruismo (RS3) & 71 & Normal \\
Espiritualidad (R4) & 76,4 & Normal \\
Motivación de interés & 82 & Alto \\
Motivación a la tarea & 83 & Alto
\end{tabular}




\begin{tabular}{lcc}
$\begin{array}{l}\text { Reconoce y utiliza las fortalezas y capacidades } \\
\text { desarrolladas. }\end{array}$ & 82,5 & Normal \\
$\begin{array}{l}\text { Aceptar la necesidad de cultivar los menos } \\
\text { obvios en su comportamiento común }\end{array}$ & 82,4 & Normal \\
$\begin{array}{l}\text { Categoría global } \\
\text { Disposición }\end{array}$ & 81 & Normal \\
\hline
\end{tabular}

Fuente: elaboración propia.

No se observaron diferencias significativas para todas las fortalezas con probabilidades entre P (0.15) y P (0.13), en la escala de resiliencia total (tabla 3). La subescala control no presentó diferencias significativas en los valores promedios de las fortalezas humildad y espiritualidad. Sin embargo, se observaron diferencias significativas en el resto de fortalezas con probabilidades entre $\mathrm{P}(0000)$ y $\mathrm{P}(0.034)$. En el grupo con puntaje mayor fueron estadísticamente inferiores a los del grupo $\geq 13,87$ puntos. De otra parte, existe correlación moderada entre factor autoestima y factor altruismo $(\mathrm{r}=0.69 / \mathrm{p}<0.01)$ y el factor familia $(\mathrm{r}=0.710 / \mathrm{p}<0.01)$. También hay una relación baja, pero significativa, con el factor filiación $(\mathrm{r}=0.352 / \mathrm{p}<.01)$. Hay correlaciones moderadas en motivación de interés, motivación al esfuerzo $(\mathrm{r}=0.66 / \mathrm{p}<0.01)$ y el factor de motivación a la tarea $(\mathrm{r}=0.61 / \mathrm{p}<0.01)$. A mayor interés mayor su capacidad. Mientras el alumno se acepte y lo motiven, se influye positivamente en él. Hay correlaciones bajas entre seguridad personal con familia $(\mathrm{r}=0.279 / \mathrm{p}<0.01)$, la autoestima $(\mathrm{r}=0.37 / \mathrm{p}<0.01)$ y el altruismo $(\mathrm{r}=0.301 / \mathrm{p}<0.01)$.

\section{Discusión}

Autores de psicología social, como Mattelart (2003) - heredero de una larga tradición de teóricos como Doob y Allport-, consideran que la actitud es la responsable de conducir la conducta. En algunos casos se establecen relaciones de congruencia, mientras que en otros se identifican diferencias considerables en cuanto a lo que alguien cree y lo que termina haciendo una vez que está inmerso en una determinada situación social. Los estudiantes exitosos presentan puntuaciones de normales a altas, en la totalidad de las fortalezas evaluadas. La fortaleza autopercibida con el valor más alto es la esperanza, seguida de gratitud, bondad, honestidad y equidad. Estas son las cinco fortalezas principales en los estudiantes. Las fortalezas con puntuaciones medias más bajas corresponden a autorregulación y prudencia. Se observa multiplicidad de caracteres individuales en perfil de fortalezas, pues cada sujeto las desarrolla de acuerdo con su historia personal y su relación con el entorno, por lo que necesariamente el contexto y las características sociodemográficas de cada uno deben tenerse en cuenta (Zemelman, 2010).

En la composición de los conglomerados se determinan dos actitudes predominantes. La primera revela cómo la preocupación empática puede ser fuente de tensión y sufrimiento para los alumnos, quienes describen que cuando ven a sus compañeros afectados por algún conflicto o problema se sienten incómodos y no saben cómo responder. La segunda se enfoca en la toma de perspectiva, es decir el educando considera que puede permanecer neutral en medio de las discusiones o enfrentamientos y detenerse para tener en cuenta las opiniones personales de sus pares.

Estas dos actitudes que sobresalen dictan en gran medida los comportamientos de los 
estudiantes que comparten posiciones encontradas, ya que una parte de ellos no se han sentido engañados o excluidos, mientras que otros, en sus comentarios, destacan que se meten con ellos cuando les recuerdan a los profesores los exámenes. También resaltan que reciben reproches cuando algo no va bien y obtienen una mala nota (E-102), y cuando preguntan en clase (E-58).

Se identifica que los estudiantes exitosos en matemáticas desarrollan comportamientos que les permiten adaptarse a situaciones imprevistas, ser poseedores de una visión positiva del futuro y flexibles para adaptar a situaciones nuevas. Tienen capacidad para promover su propio bienestar y habilidades para actuar conforme a lo que han decidido hacer con su vida con influencias positivas en la vida familiar y, asocia a ello, la espiritual.

En general, según García (2008), el éxito escolar tiene una naturaleza multidimensional en la que participan elementos cognitivos y emocionales. En este sentido, los alumnos conforman su disposición y comportamiento según la aceptación de su entorno académico (Castanyer, 2010). Ante esto se observa que los estudiantes tienen dudas, ya que aquellos con un desempeño normal o deficiente son los que tienden a sentirse más cómodos en la escuela, mientras que los que tienen un desempeño excelente son los que son más críticos sobre cómo deben editar su personalidad y sus conductas para complacer. En esa perspectiva señalan: "trabajo solo si me va bien; si no, normal" (E890); "somos indiferentes a los comentarios negativos o felicitaciones, nos enfocamos en aprender más y más" (E457); "me gusta que me vaya bien. No presto atención a nada" (E367); "participo indiferente a lo que los demás piensen” (E901).

Los resultados señalan que los estudiantes de menor edad tienen mayores niveles de resiliencia total, aceptación positiva del cambio y control. No obstante, los estudiantes de cuarto semestre asumen el reto de iniciar acciones, solicitar incentivos, vincularse a proyectos, hablar con docentes, y ponen en marcha todas sus capacidades como seres humanos. Se adaptan a los cambios, asumen responsabilidades y utilizan recursos sociales, morales y espirituales para el desenvolvimiento exitoso en su nueva vida (Celis et al., 2001). Las notas en las tarjetas muestran una resistencia significativa por parte de los estudiantes de alto rendimiento para formar nuevos lazos y relaciones. Por otro lado, aquellos que tienen problemas con las calificaciones son los más expresivos y tienden a tener no más de dos amigos. Otro de los comportamientos que se observan en la convivencia es la superioridad -o egocentrismo - con la que los alumnos pueden responder cuando sus compañeros los molestan. En esta línea, expresan: "me gusta saber más que los demás y entender siempre lo que está pasando" (E021).

La responsabilidad por su buen desempeño también genera una presión social en la que sus dificultades en algunas áreas, como la educación física o artística, estimulan comentarios negativos en los demás alumnos del grupo. Cuando son juzgados, se muestran retraídos; se cierran cada vez más a su círculo social más cercano - formado por uno o dos-; y aumentan uno de sus comportamientos característicos, el triunfador y estable, ya que no les gustan los cambios y reflejan gran desconfianza. La oscilación entre las dimensiones sobresalientes del sufrimiento y la perspectiva revela, entre otras cosas, que estos jóvenes se encuentran aún en una etapa crucial para formar su personalidad social. En ese sentido, aunque sus impulsos básicos los incitan a cuidar o ayudar, no saben cómo dejar de sentirse 
abrumados y optan por no arriesgar, fingir desinterés o simple indiferencia hacia el sujeto.

\section{Conclusiones}

Los factores asociados a la resiliencia en estudiantes universitarios fueron: aceptación positiva del cambio, competencia personal, control y espiritualidad; generadas a partir del análisis factorial de las respuestas obtenidas mediante la aplicación del test. Los estudiantes de menor edad y de semestres inferiores muestran valores significativamente más altos en la resiliencia total, al igual que en la subescala aceptación positiva al cambio.

La resiliencia se relaciona significativamente con todas las fortalezas del carácter, así como con la aceptación positiva al cambio y la competencia personal, a excepción de la prudencia. En la investigación se identifica que, si bien la resiliencia les ayuda a ser más tolerantes y a comprender mejor lo que sucede en el entorno, comportamientos externos los alejan de su enfoque, sus valores y su personalidad. Es precisamente en esos desafíos donde se manifiestan las fortalezas que les brindan un alto desempeño educativo.

Los estudiantes exitosos cuentan con niveles de resiliencia de normal a alto. Presentan diferencias en su motivación de logro, interés y esfuerzo, en comparación con el promedio de estudiantes menos exitosos. Su motivación es interna y es generada a través de su esfuerzo. Quienes estudian en ambientes vulnerables cuentan con características que les ayudan a vivir y a afrontar sus condiciones de vida.

Los estudiantes de diferentes programas académicos y exitosos en matemáticas demuestran habilidades de pensamiento formal, pensamiento creativo, aplicación de la matemática y resiliencia, que inciden en su calidad de vida. Cada estudiante reconoce y utiliza sus fortalezas y capacidades desarrolladas, al aceptar la necesidad de cultivar las menos obvias en su comportamiento común.

Con referencia a las relaciones interpersonales entre estudiantes, el clima de convivencia a nivel general es bueno, pero, cuando se enfoca la atención en los salones de clase, surgen inconsistencias como: la intolerancia de los estudiantes frente a los compañeros con resultados académicos superiores; la soledad que experimentan los estudiantes de buen rendimiento cuando quieren participar en diferentes proyectos académicos; además de las divisiones y brechas que genera la débil integración entre los educandos que conforman cada grupo escolar.

A partir de las experiencias diarias de convivencia, se destacan algunas actitudes de violencia verbal usadas para ridiculizar o amedrentar a quienes algunos jóvenes consideran diferentes a ellos. Entre ellas hay palabras de jerga juvenil o gritos sin fundamento. Entre tanto, la respuesta de los estudiantes que ocupan los primeros lugares es invisibilizar sus acciones y decisiones, con el propósito de pasar inadvertidos y dejar de ser objeto de atención por parte de los docentes y estudiantes, una acción que no solo afecta a la institución, sino al proceso de desarrollo del educando.

Finalmente, la capacidad que pueda tener el desarrollo excepcional para resolver conflictos y cerrar las brechas que afectan las dinámicas de convivencia en las instituciones educativas no ha sido direccionada en ese sentido dentro de cada una, sino que su presencia ha sido 
asumida como un factor más dentro del engranaje social que posibilita el funcionamiento de la comunidad educativa.

La decisión de abordar la excelencia en el rendimiento académico y su incidencia en el clima de las relaciones escolares desvela que este tema no ha sido tratado de forma prioritaria por las instituciones educativas y que a partir de eso se han fortalecido comportamientos de intolerancia, exclusión e inseguridad, que no solo desfavorecen el presente y el futuro del estudiante, sino que también desestabilizan el cumplimiento de la misión educativa establecida en el proyecto educativo institucional.

\section{Referencias}

Castanyer, O. (2010). Assertiveness: expression of a healthy self-esteem (31 st edition). Bilbao: Desclée de Brower.

Castro, A. (2010). Concepciones teóricas acerca de la Psicología Positiva. En A. Castro (Comp.), Fundamentos de Psicología Positiva (pp. 17-41). Buenos Aires: Paidós.

Celis, J., Bustamante, M., Cabrera, D., Cabrera, M., Alarcón, W., \& Monge, E. (2001). Ansiedad y estrés académico en estudiantes de medicina humana del primer y sexto año. Anales de la Facultad de Medicina, 62(01), 25-30.

Covington, M. (2000). Goal Theory, Motivation, and School Achievement: An Integrative Review. Annual Review of Psychology, 51, 171-200.

Crombie, P., López, M., Mesa, M., \& Samper, L. (2015). Adaptación de la Escala de Resiliencia de Wagnild y Young. Bogotá: Universidad de Los Andes.

Cruz, M. (2016). Factores que influyen en el rendimiento académico del estudiante. Escenarios: empresa y territorio. 5(5), 93-128.

Duek, C. (2010). Childhood, development and knowledge: children and their socialization. Latin American Journal of Social Sciences, Children and Youth, 8, 799-808.

Gallardo, H., Vergel, M., \& Villamizar, F. (2018). Investigación intervención y enfoque multimétodo en ciencias humanas y educación matemática. Logos Ciencia y Tecnología, 9(2), 84-96. https://doi.org/10.22335/rlct.v9i2.458

García, F. (2008). Motivate for learning from the orienting activity. Madrid: CIDE.

García, L. (2003). La psicología positiva: del modelo de la reparación al modelo del fortalecimiento. Revista Hojas Informativas de los Psicólogos de las Palmas, 56, 1-5.

Grotberg, E. (2006). ¿Qué entendemos por resiliencia? ¿Cómo promoverla? ¿Cómo utilizarla?. En E. Grotberg (Comp.), La resiliencia en el mundo de hoy. Como superar las adversidades (pp. 17-57). España: Gedisa, S. A.

Hallinger, P., \& Heck, R. (2014). Collaborative leadership and school improvement: Understanding the impact on school capacity and student learning. REICE. IberoAmerican Journal on Quality, Efficacy and Change in Education, 12(4), 71-88. https://doi. org/10.1080/13632431003663214

Jadue, G. (2002). Factores Psicológicos que predisponen al bajo rendimiento, al fracaso y a la deserción escolar. Estudios Pedagógicos, 28, 193-204. 
Kotliarenco, M. (1999). Algunas particularidades metodológicas en los estudios sobre Resiliencia. Chile: MAK Consultores.

Kotliarenco, M., Cáceres, I., \& Fontecilla, M. (1997). Estado de Arte en Resiliencia. Washington, D.C.: Organización Panamericana de la Salud.

Mata, S., Gómez, M., \& Calero M. (2018). Resolución de problemas Interpersonales en Niños en Exclusión Social. Revista Latinoamericana de Psicología, 52(2), 107-116. http:// dx.doi.org/10.14349/rlp.2018.v50.n2.4

Mattelart, A. (2003). Interview with Armand Mattelart. Information society and intellectual project. Sign and Thought Magazine, 22(43), 71-77.

Moreno, A. (2011). Educación y violencia en la Venezuela actual. Revista de Pedagogía, 32(90), 14-18.

Olivera, J., Braun, M., \& Roussos, A. (2011). Instruments for the Evaluation of Empathy in Psychotherapy. Argentine Journal of Clinical Psychology, 20, 121-132.

Osorio, M., Mejía, L., \& Navarro, J. (2009). Factores psicosociales que influyen en el éxito o fracaso del aprovechamiento escolar en la asignatura de física básica. Espacios Públicos, 12(26), 261-276.

Pérez, Á. (2012). Ethnography as an integrative method. Colombian Journal of Psychiatry, 41(2), 421-428. https://doi.org/10.1016/S0034-7450(14)60015-9

Rivera, D., Gallardo, H., Mora, A., Gómez, L., \& Barrios D. (2018). Rendimiento académico y relaciones intrafamiliares en estudiantes repitentes en secundaria. En S. Carrillo, B. Sanabria, V. Bermúdez, \& J. Espinosa, Actores en la Educación: una Mirada desde la Psicología Educativa (pp. 181-208). Barranquilla: Ediciones Universidad Simón Bolívar.

Rodríguez, C., \& Valdivieso A. (2008). El éxito escolar de alumnos en condiciones adversas. Revista Latinoamericana de Estudios Educativos, (1-2), 81-106.

Saavedra, E. (2003). El enfoque cognitivo procesal sistémico, como posibilidad de intervenir educativamente en la formación de sujetos resilientes. Valladolid: Universidad de Valladolid.

Sánchez, D., \& Robles, M. (2015). Escala de Resiliencia 14 ítems (RS-14): Propiedades Psicométricas de la Versión en Español. Revista Iberoamericana de Diagnóstico y Evaluación, 40, 103-113.

Sierra, R. (1989). Social research techniques, theory and exercises (8th ed.). España: Paraninfo S. A.

Wagnild, G., \& Young, H. (1993). Development and psychometric evaluation of the resilience scale. Journal of Nursing Measurement, 1(2), 165-178.

Wagnild, G., \& Young, H. (2015). ER. Escala de Resiliencia. Barcelona: Draft Grupo Editorial.

Zemelman, H. (2010). Sujeto y subjetividad: la problemática de las alternativas como construcción posible. Polis Revista Latinoamericana, 27, 1-12. 